\title{
SPREADABLE ARRAYS AND MARTINGALE STRUCTURES
}

\author{
B. GAIL IVANOFF and N. C. WEBER ${ }^{\complement}$
}

(Received 20 November 2003; revised 22 January 2004)

Communicated by V. T. Stefanov

\begin{abstract}
Kallenberg has introduced the concept of conditional spreadability for random sequences and has developed characterizations of this property in terms of one dimensional martingales and optional times, and as well has proven a predictable sampling theorem. This paper investigates the relationship between planar martingale structures and the natural analogues of conditional spreadability when extended to arrays of random elements. Analogues of the predictable sampling theorem are also established for spreadable arrays.
\end{abstract}

2000 Mathematics subject classification: primary 60G09.

Keywords and phrases: spreadable array, contractable array, exchangeable array, martingale, adapted time, predictable sampling.

\section{Introduction}

The concept of spreadability of finite and infinite sequences of random elements has been studied by many authors, including Ryll-Nardzewski [11], Kingman [9] and Kallenberg ([5, 7]). A sequence is said to be exchangeable if its distribution is invariant under permutations that leave all but a finite number of elements fixed whereas a sequence is said to be spreadable if all subsequences of the same size have the same distribution.

In [7] Kallenberg has related spreadability of sequences to a filtration $\mathscr{F}$ to which the sequence is adapted, and has linked the concepts of $\mathscr{F}$-spreadability and $\mathscr{F}$-martingales. In light of the richness and diversity of the theory of multidimensional martingales (see, for example, Merzbach [10]), the goal of this work is to explore the

The first author was supported by a grant from the Natural Sciences and Engineering Research Council of Canada.

(C) 2005 Australian Mathematical Society $1446-7887 / 05 \$ A 2.00+0.00$ 
connections between these two structures in the context of arrays of random elements. We shall see that the various natural two-dimensional analogues of the concept of spreadability give rise to different martingale structures on the plane.

In the case of infinite sequences, Ryll-Nardzewski [11] showed that spreadability and exchangeability are in fact equivalent, so by de Finetti's Theorem an infinite spreadable sequence is mixed i.i.d. Kingman [9] noted that the two structures are not equivalent for finite sequences. While finite echangeable sequences can be represented as mixtures of 'urn' sequences (see, for example, Aldous [2]), no such characterization exists for finite spreadable sequences.

Extending the idea of spreadability to arrays leads to two different approaches. For the stronger, separate spreadability, we select a subsequence of column indices and a separate subsequence of row indices to determine the elements that form a subarray. For the weaker, joint spreadability, we apply the same subsequence of indices to both rows and columns to form the subarray. (See [6] and [4].)

For a sequence, when spreadability is related to a filtration $\mathscr{F}$, we shall see that there are martingale characterizations of $\mathscr{F}$-spreadability in terms of either a translation operator or a deletion operator. Although the two operators are essentially equivalent for sequences, this is no longer the case for arrays. Translations lead naturally to weaker definitions of conditional spreadability than do deletions.

In the case of arrays, conditional exchangeability was defined and characterized in Ivanoff and Weber [4]. In light of the preceding discussion, combining the different approaches to conditional spreadability for arrays gives rise to four different structures, each of which will be shown to have its own martingale characterization.

Further, new predictable sampling theorems will be established for each of the conditional spreadable array structures. In some cases, one-dimensional technology can be applied to establish these results, but when the sampling is done using times that are predictable in a two-dimensional sense, new techniques are required.

\section{Notation and definitions}

A sequence of random elements $\left(X_{1}, \ldots, X_{n}\right)(n$ may be $\infty)$ taking their values in some measurable space $(G, \mathscr{G})$ is spreadable if for any finite $m<n$ and increasing subsequence of indices $k_{1}<\cdots<k_{m}$,

$$
\left(X_{k_{1}}, \ldots, X_{k_{m}}\right)=_{\mathscr{D}}\left(X_{1}, \ldots, X_{m}\right) .
$$

Kallenberg has suggested that 'contractable' is a more suitable name for this property. From [7] we note that when $n<\infty$, (1) is equivalent to the condition

$$
\phi_{k} \circ X:=\left(X_{1}, \ldots, X_{k-1}, X_{k+1}, \ldots, X_{n}\right)=\mathscr{D}\left(X_{1}, \ldots, X_{n-1}\right)=\phi_{n} \circ X, \forall k<n .
$$


We shall refer to $\phi_{k}$ as the deletion operator. By induction, it is easily seen that (2) implies (1) and so the behaviour of the contracted sequence after deletion of one or more elements determines the spreadability property. To extend (2) to infinite sequences, we see that (1) is equivalent to the condition that for $n$ finite or infinite and any finite $k, j, 1 \leq k, j \leq n$,

$$
\phi_{k} \circ X=\mathscr{D} \phi_{j} \circ X .
$$

If (1) is true for any finite subset of distinct (but not necessarily increasing) indices, then the sequence is exchangeable.

As in the case of exchangeability, conditional spreadability for sequences is defined with respect to a discrete filtration $\mathscr{F}=\left(\mathscr{F}_{0}, \mathscr{F}_{1}, \ldots\right)$. We say that the sequence $\left(X_{1}, \ldots, X_{n}\right)$ is $\mathscr{F}$-spreadable if it is adapted to $\mathscr{F}$ and when conditioned on $\mathscr{F}_{k}$, $\left(X_{k+1}, \ldots, X_{n}\right)$ is spreadable for every $k$. This property can be expressed in two equivalent ways: first,

$$
\text { conditioned on } \mathscr{F}_{k}, \theta_{k} \circ X \text { is spreadable, } \forall k<n \text {, }
$$

where $\theta_{k} \circ X$ is the shifted sequence $\left(X_{k+1}, \ldots, X_{n}\right)$. As in [7], to avoid the requirement of the existence of regular conditional distributions, we interpret (4) in terms of conditional probabilities given any set $A \in \mathscr{F}_{k}$ with $P(A)>0$. It is trivially true that any spreadable sequence is $\mathscr{F}$-spreadable with respect to the minimal filtration generated by the sequence.

Using (3), we see that (4) is equivalent to the following:

$$
\phi_{h} \circ X={\mathscr{D} \mid \mathscr{F}_{k}}_{\boldsymbol{1}} \phi_{j} \circ X, \forall k<h, j \leq n ; k, h, j<\infty
$$

where ' $=\mathscr{Q} \mid \mathscr{F}_{k}$ ' indicates equality in distribution, given $\mathscr{F}_{k}$.

We shall see that the two dimensional analogue of (5) is strictly stronger than the analogue of (4).

For clarity and notational convenience in what follows, given a $c \times r$ array we shall generally assume that either both $c, r<\infty$ or $c=r=\infty$. However, all definitions and theorems can be adapted in a straightforward way to include the case in which only one of $c$ and $r$ is finite.

Let $X=\left(X_{i j}: 1 \leq i \leq c, 1 \leq j \leq r\right)$ be a $c \times r$ array of random elements taking their values in some measurable space $(G, \mathscr{G}) . X$ is separately spreadable (SS) if for all $m<c, q<r ; m, q<\infty$ and $i_{1}<\cdots<i_{m}, j_{1}<\cdots<j_{q}$,

$$
\left(X_{i_{k} j_{h}}: 1 \leq k \leq m, 1 \leq h \leq q\right)=_{\mathscr{D}}\left(X_{i j}: 1 \leq i \leq m, 1 \leq j \leq q\right) .
$$

In analogy to (3), $X$ is SS if and only if for every $h, k \leq c$ and $l, m \leq r ; h, k$, $l, m<\infty$,

$$
\phi_{h .} \circ X=\mathscr{D} \phi_{k} \circ X \text { and }
$$




$$
\phi_{\cdot l} \circ X=\mathscr{D} \phi_{\cdot m} \circ X
$$

where $\phi_{h} \circ X$ is the array $X$ with column $h$ deleted, and $\phi_{\cdot l} \circ X$ is the array $X$ with row $l$ deleted. Note that $\phi_{h}$ and $\phi_{. l}$ commute and denote $\phi_{h l}=\phi_{h .} \circ \phi_{. l}$.

The square array $X=\left(X_{i j}: 1 \leq i, j \leq n\right)(n$ may be $\infty)$ is jointly spreadable (JS) if for all finite $m<n$ and $i_{1}<\cdots<i_{m}$,

$$
\left(X_{i_{k} i_{h}}: 1 \leq k, h \leq m\right)=_{\mathscr{D}}\left(X_{i j}: 1 \leq i, j \leq m\right) .
$$

Equivalently, $X$ is JS if and only if for every $j, k \leq n ; j, k<\infty$,

$$
\phi_{j j} \circ X=\mathscr{D} \phi_{k k} \circ X
$$

There is an extensive literature on exchangeable and related arrays which has grown from the seminal works of Aldous [1] and Hoover [3] that extended de Finetti's theorem to infinite arrays with appropriate symmetries. Aldous [2] is an excellent general reference on exchangeability. Similar representations for infinite spreadable arrays have been established by Kallenberg [6]. The following characterizations are developed without reference to these representations and the techniques used here apply to both finite and infinite arrays.

In order to define conditional spreadability for arrays, we assume that we are given an arbitrary filtration $\mathscr{F}=\left(\mathscr{F}_{i j}: 0 \leq i \leq c, 0 \leq j \leq r\right)$ to which $X$ is adapted (that is, $\mathscr{F}_{i j} \subseteq \mathscr{F}_{h k}$ if $i \leq h$ and $j \leq k$, and $X_{i j}$ is $\mathscr{F}_{i j}$-measurable, $\forall i, j$ ). Roughly speaking, we will say that an array has a particular property in the weak $\mathscr{F}$ sense if, conditioned on $\mathscr{F}_{j k}$, the translated array $\theta_{j k} \circ X:=\left(X_{h l}: j+1 \leq h \leq c, k+1 \leq l \leq r\right)$ has the required property for all $(j, k) \in\{1, \ldots, c\} \times\{1, \ldots, r\}$ (cf. (4)). On the other hand (cf. (5)), suppose that the actions defining the property in question are carried out in such a way that the first $j$ columns and $k$ rows are left unchanged and consider the conditional distribution of the entire array given $\mathscr{F}_{j k}$. Then we say that the array has the property in the strong $\mathscr{F}$ sense if the conditional distributions of the new array and the original are the same for all $(j, k) \in\{1, \ldots, c\} \times\{1, \ldots, r\}$. Figures 1 (a) and (b) illustrate the fundamental difference between the various weak $\mathscr{F}$ - and strong $\mathscr{F}$-properties. As can be seen, when conditioning on $\mathscr{F}_{j k}$, the weak $\mathscr{F}$-properties involve only the conditional distribution of the shifted array $\theta_{j k} \circ X$ indicated by the shaded region in Figure 1 (a), whereas the shaded region in Figure 1 (b) illustrates that the strong $\mathscr{F}$-properties involve the conditional distribution of the entire shell outside ( $X_{h l}: 1 \leq h \leq j, 1 \leq l \leq k$ ), which includes the shoulder regions $S_{1}$ and $S_{2}$, that is, the set of variables in the first $k$ rows that are not in the first $j$ columns, or in the first $j$ columns but not in the first $k$ rows.

With these concepts in mind, we define the various conditional spreadability properties as follows. 


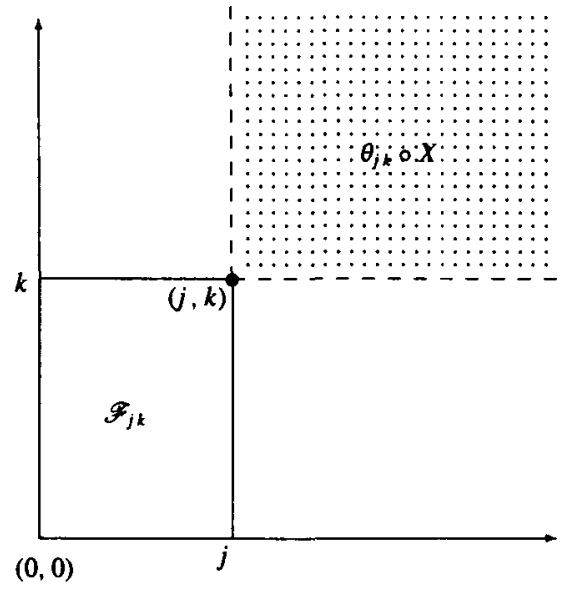

(a) The weak $\mathscr{F}$-property

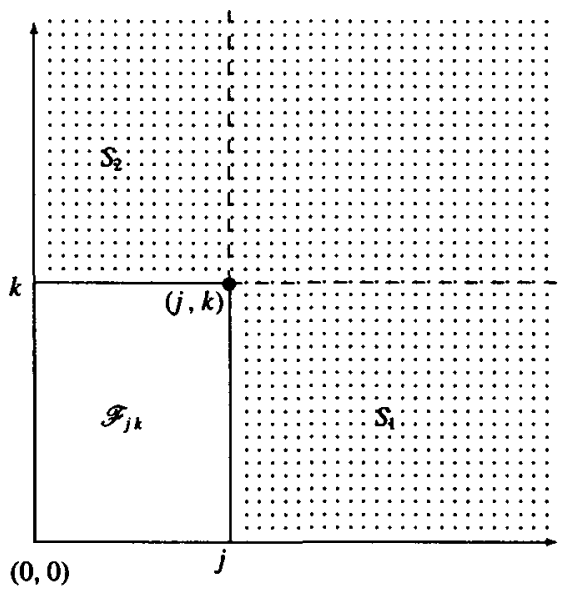

(b) The strong $\mathscr{F}$-property

FIGURE 1. The weak and strong $\mathscr{F}$-properties.

We call $X$ weak $\mathscr{F}$-SS if (cf. (4))

$$
\text { conditioned on } \mathscr{F}_{j k}, \theta_{j k} \circ X \text { is SS, } \forall j<c \text { and } \forall k<r \text {. }
$$

Similarly, the $n \times n$ array $X$ is weak $\mathscr{F}$-JS if

$$
\text { conditioned on } \mathscr{F}_{k k}, \theta_{k k} \circ X \text { is JS, } \forall k<n \text {. }
$$

We will say that $X$ is strong $\mathscr{F}$-SS if (cf. (5), (7), (8)) for any $i<h, k \leq c$ and $j<l, m \leq r ; h, k, l, m<\infty$,

$$
\begin{aligned}
& \phi_{h .} \circ X=\mathscr{D} \mid \mathscr{F}_{i j} \phi_{k} \circ X \quad \text { and } \\
& \phi_{. l} \circ X=\mathscr{D F}_{i j} \phi_{\cdot m} \circ X .
\end{aligned}
$$

The $n \times n$ array $X$ is strong $\mathscr{F}$-JS if (cf. (5), (10)) for every $i<j, k \leq n ; j, k<\infty$,

$$
\phi_{j j} \circ X=\mathscr{D} \mid \mathscr{F}_{i i} \phi_{k k} \circ X \text {. }
$$

It is clear that the strong $\mathscr{F}$-properties imply the weak $\mathscr{F}$-properties, and they are equivalent when $\mathscr{F}$ is the minimal filtration generated by the array (since in this case, $X$ is separately (respectively, jointly) spreadable if and only if the corresponding strong $\mathscr{F}$ - and weak $\mathscr{F}$-properties hold). For general filtrations, the converse is not true: an example is given in [4, page 350$]$ that is weak $\mathscr{F}$-SS but not strong $\mathscr{F}$-SS. We repeat it here, denoting the $i$ th column of an array $X$ by $X_{i}$. and the $j$ th row of $X$ by $X_{, j}$. 
EXAMPLE 2.1. Let $\left\{\xi_{i}\right\},\left\{\eta_{j}\right\}$ and $\left\{\lambda_{i j}\right\}$ be independent $U(0,1)$ random variables. Let $X_{i j}=\xi_{i} \eta_{j} \lambda_{i j}$ and $\mathscr{F}_{i j}=\sigma\left\{\xi_{k}, k \leq i ; \eta_{l}, \lambda_{h l}, l \leq j, h \geq 1\right\}$ for $i, j \geq 1$. Given

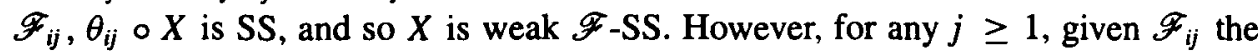
columns $X_{i+1}, X_{i+2}, \ldots$ are not identically distributed, and so $X$ is not strong $\mathscr{F}_{\text {-SS. }}$

For simplicity in the following sections, we shall refer to the strong $\mathscr{F}$ properties simply as $\mathscr{F}$-SS or $\mathscr{F}$-JS when no ambiguity arises.

Both 1- and 2-dimensional martingale structures and random times will be used in the sequel. Given the filtration $\mathscr{F}=\left(\mathscr{F}_{i j}: 0 \leq i \leq c, 0 \leq j \leq r\right)$ denote the (one-dimensional) filtrations $\mathscr{F}^{1}=\left(\mathscr{F}_{i}^{1}: 1 \leq i \leq c\right)$ and $\mathscr{F}^{2}=\left(\mathscr{F}_{j}^{2}: 1 \leq j \leq r\right)$, where $\mathscr{F}_{i}^{1}=\bigvee_{j=1}^{r} \mathscr{F}_{i j}$ and $\mathscr{F}_{j}^{2}=\bigvee_{i=1}^{c} \mathscr{F}_{i j}$. An integrable process

$$
M=\left\{M_{i j}: 0 \leq i \leq c, 0 \leq j \leq r\right\}
$$

adapted to a filtration $\mathscr{F}$ is an (F्F-) martingale if for all $i, j, h, k$ such that $i \leq h$, $j \leq k, E\left[M_{h k} \mid \mathscr{F}_{i j}\right]=M_{i j}$ a.s. $M$ is a 1 -martingale if for all $i, j, h$ such that $i \leq h$, $E\left[M_{h j} \mid \mathscr{F}_{i}^{1}\right]=M_{i j}$ a.s. Analogously, $M$ is a 2-martingale if for all $i, j, k$ such that $j \leq k, E\left[M_{i k} \mid \mathscr{F}_{j}^{2}\right]=M_{i j}$ a.s.

The random vector $(S, T)$ taking values in $\mathbb{N}^{2}$ is an adapted random time with respect to $\mathscr{F}$ if $\{S=i, T=j\} \in \mathscr{F}_{i j}$, for all $i, j \geq 1$. It is a predictable random time if $\{S=i, T=j\} \in \mathscr{F}_{i-1, j-1}$, for all $i, j \geq 1$. Similarly, the $\mathbb{Z}_{+}$-valued random variable $S$ is an $\mathscr{F}^{1}$-adapted (predictable) random time if $\{S=i\} \in \mathscr{F}_{i}^{1}\left(\mathscr{F}_{i-1}^{1}\right)$, for all $i \geq 1 . \mathscr{F}^{2}$-adapted and predictable random times are defined similarly. Note that if $(S, T)$ is $\mathscr{F}$-adapted (predictable), then $S$ and $T$ are $\mathscr{F}^{1}$ - and $\mathscr{F}^{2}$-adapted (predictable), respectively. However, in general the converse is not true. (Note that we have avoided use of the more standard term of stopping or optional time due to the fact that two dimensional stopping times can be defined in various ways.)

\section{Separate spreadability}

3.1. Strong $\mathscr{F}$-separately spreadable arrays Recall that an array $X$ is strong $\mathscr{F}$-SS, or just $\mathscr{F}$-SS, if and only if for every $i<h, k \leq c$ and $j<l, m \leq r$; $h, k, l, m<\infty$,

$$
\phi_{h \cdot} \circ X=\mathscr{D}_{\mid F_{i}} \phi_{k} \circ X \text { and } \phi_{\cdot l} \circ X=\mathscr{D}_{\mid \mathscr{F}_{i}} \phi_{\cdot m} \circ X
$$

The fact that $j$ is arbitrary in the first equation above and $i$ in the second yields a very simple lemma that will allow us to apply known results for spreadable sequences to SS arrays. 
LEMMA 3.1. Let $X$ be a finite or infinite array. $X$ is $\mathscr{F}$-SS if and only if the sequence of column vectors $\left(X_{i} ; 1 \leq i \leq c\right)$ is $\mathscr{F}^{1}$-spreadable and the sequence of row vectors $\left(X_{, j} ; 1 \leq j \leq r\right)$ is $\mathscr{F}^{2}$-spreadable.

PROOF. We shall consider only the column vectors as the proof for rows is identical. Assume that $X$ is $\mathscr{F}$-SS. We must show that for $i<h, k \leq c ; i, h, k<\infty$,

$$
\phi_{h} \circ\left(X_{1}, X_{2}, \ldots\right)=_{\mathscr{D} \mid F_{i}^{1}} \phi_{k} \circ\left(X_{1}, X_{2}, \ldots\right) \text {. }
$$

As pointed out in Section 1, we interpret (15) in terms of elementary conditional probabilities given any set $A \in \mathscr{F}_{i}^{1}$ with $P(A)>0$. Therefore, (15) is an immediate consequence of (13), since it is sufficient to check equality of conditional probabilities on $\bigcup_{j=1}^{r} \mathscr{F}_{i j}$, a $\pi$-system that generates $\mathscr{F}_{i} \mathbf{1}$.

The converse is trivial: (15) implies (13) since $\mathscr{F}_{i j} \subseteq \mathscr{F}_{i}{ }^{1}$.

Lemma 3.1 allows us to give an analogue of [7, Lemma 3.1] for SS arrays. First, the sequence $\left(X_{i} ; 1 \leq i \leq c\right)$ is $\mathscr{F}^{1}$-stationary if $\theta_{\tau} \circ\left(X_{1}, X_{2}, \ldots\right)=\mathscr{g}\left(X_{1}, X_{2,}, \ldots\right)$ for every bounded $\mathscr{F}^{1}$-adapted time $\tau$. For $c<\infty$, this is interpreted as

$$
\left(X_{\tau+1}, \ldots, X_{\tau+k}\right)=_{\mathscr{D}}\left(X_{1}, \ldots, X_{k}\right) \quad \text { whenever } \tau+k \leq c \text { a.s. }
$$

We define $\mathscr{F}^{2}$-stationarity analogously for the row vectors of $X$.

We next define the prediction sequences

$$
\begin{aligned}
& \mu_{i .}=P\left[\theta_{i} \circ\left(X_{1 .}, X_{2 .}, \ldots\right) \in \cdot \mid \mathscr{F}_{i}^{1}\right] \\
& \mu_{. j}=P\left[\theta_{j} \circ\left(X_{.1}, X_{.2}, \ldots\right) \in \cdot \mid \mathscr{F}_{j}^{2}\right] .
\end{aligned}
$$

As before, the necessity of regularity properties of the conditional probabilities is avoided by interpreting (16)-(17) in terms of elementary conditional probabilities. We say that $\mu_{i}$. forms an $\mathscr{F}^{1}$-martingale if

$$
\theta_{i+1} \circ\left(X_{1}, X_{2,} \ldots\right)=g, \theta_{i} \circ\left(X_{1}, X_{2}, \ldots\right)
$$

over $\mathscr{F}_{i}{ }_{i}$ for all $i \geq 0$, that is, for $A \in \mathscr{F}_{i}{ }^{1}, i \geq 0$,

$$
P\left[\theta_{i+1} \circ\left(X_{1}, X_{2}, \ldots\right) \in \cdot ; A\right]=P\left[\theta_{i} \circ\left(X_{1}, X_{2}, \ldots\right) \in \cdot ; A\right] .
$$

For finite sequences, this condition is interpreted as: for $i=0, \ldots, c-2$,

$$
\left(X_{i+2}, \ldots, X_{c}\right)=_{\mathscr{D}}\left(X_{i+1}, \ldots, X_{c-1}\right) \text { over } \mathscr{F}_{i}^{1} .
$$

We define the $\mathscr{F}^{2}$-martingale condition for $\mu_{. j}$ analogously. 
We may also define truncated prediction sequences, using truncated arrays. For $j \leq r$, denote $X^{(\cdot j)}=\left(X_{i k}, 1 \leq i \leq c, 1 \leq k \leq j\right)$ and for $i \leq c$, denote $X^{(i)}=\left(X_{h j}, 1 \leq h \leq i, 1 \leq j \leq r\right)$. We define the prediction sequences

$$
\begin{aligned}
& \rho_{i j}=P\left[\theta_{i} \circ\left(X_{1 .}^{(\cdot j)}, X_{2 .}^{(\cdot)}, \ldots\right) \in \cdot \mid \mathscr{F}_{i}^{1}\right] \\
& \tilde{\rho}_{i j}=P\left[\theta_{j} \circ\left(X_{.1}^{(i \cdot)}, X_{.2}^{(i \cdot)}, \ldots\right) \in \cdot \mid \mathscr{F}_{j}^{2}\right] .
\end{aligned}
$$

The following lemma characterizes an $\mathscr{F}$-SS array in terms of martingale structures.

LEMMA 3.2. Let $X=\left(X_{i j}: 1 \leq i \leq c, 1 \leq j \leq r\right)$ be a finite or infinite $\mathscr{F}$-adapted random array in some measurable space $G$. The following are equivalent:

(1) $X$ is $\mathscr{F}$-SS.

(2) $\left(X_{i} ; 1 \leq i \leq c\right)$ is $\mathscr{F}^{1}$-stationary and $\left(X_{. j} ; 1 \leq j \leq r\right)$ is $\mathscr{F}^{2}$-stationary.

(3) $\mu_{i}$. forms an $\mathscr{F}^{1}$-martingale and $\mu_{\text {.j }}$ forms an $\mathscr{F}^{2}$-martingale.

(4) $\rho_{i j}$ forms a 1-martingale and $\tilde{\rho}_{i j}$ forms a 2-martingale.

PROOF. The equivalence of conditions (1)-(3) above is an immediate consequence of [7, Lemma 3.1] and Lemma 3.1. The equivalence of (3) and (4) follows from the simple observation that the law of an array is determined by the laws of the truncated arrays.

A martingale characterization for spreadability of sequences can be expressed in terms of deletion operators. First, we observe that for a sequence $X=\left(X_{i} ; 1 \leq i \leq n\right)$ of random elements adapted to a filtration $\mathscr{F}$,

$$
\phi_{i} \circ\left(X_{1}, X_{2}, \ldots\right)=\left(X_{1}, \ldots, X_{i-1}, \theta_{i} \circ\left(X_{1}, X_{2}, \ldots\right)\right) .
$$

Defining $v_{k}=P\left[\phi_{k+1} \circ X \in \cdot \mid \mathscr{F}_{k}\right]$, we obtain Lemma 3.3 below, which gives an alternative martingale characterization to [7, Lemma 3.1].

LEMMA 3.3. Let $X=\left(X_{i} ; 1 \leq i \leq n\right)$ be a finite or infinite sequence of random elements adapted to a filtration $\mathscr{F}$. The following are equivalent:

(1) $X$ is $\mathscr{F}$-spreadable.

(2) For any $\mathscr{F}$-predictable bounded random time $\tau$ such that $\tau \leq m<\infty$ a.s., $\phi_{\tau} \circ X=\mathscr{D} \phi_{m} \circ X$.

(3) $v_{k}$ is an $\mathscr{F}$-martingale.

The proof of this lemma is easily adapted from the proof of Lemma 4.1 in Section 4. In light of Lemma 3.3, the martingale characterizations in Lemma 3.2 can be restated in terms of deletion operators.

We next extend the concept of optional skipping or predictable sampling to SS arrays, and we shall do so in terms of both 1- and 2-dimensional random times. 
THEOREM 3.4. Let $X$ be a finite or infinite $\mathscr{F}$-SS array.

(1) Let $S_{1}<S_{2}<\cdots<S_{i}$ be finite $\mathscr{F}^{1}$-predictable times in $\{1, \ldots, c\}$ and let $T_{1}<T_{2}<\cdots<T_{j}$ be finite $\mathscr{F}^{2}$-predictable times in $\{1, \ldots, r\}$. Then

$$
\begin{aligned}
& \left(X_{S_{1}}, \ldots, X_{S_{i}}\right)=\mathscr{D}\left(X_{1}, \ldots, X_{i .}\right) \text { and } \\
& \left(X_{T_{1}}, \ldots, X_{. T_{j}}\right)=\mathscr{D}\left(X_{.1}, \ldots, X_{. j}\right) .
\end{aligned}
$$

(2) Let $S_{1}<S_{2}<\cdots<S_{i}$ be finite $\mathscr{F}^{1}$-predictable times in $\{1, \ldots, c\}$ and let $T_{1}<T_{2}<\cdots<T_{j}$ be finite $\mathscr{F}^{2}$-predictable times in $\{1, \ldots, r\}$ and suppose that one of the following two conditions is satisfied:

(a) $S_{h}$ is $\mathscr{F}_{0}^{2}$-measurable, for all $h=1, \ldots$, i or $T_{k}$ is $\mathscr{F}_{0}^{1}$-measurable, for all $k=1, \ldots, j$.

(b) For all $h=1, \ldots, i$ and $k=1, \ldots, j,\left(S_{h}, T_{k}\right)$ are $\mathscr{F}$-predictable times in $\{1, \ldots, c\} \times\{1, \ldots, r\}$.

Then

$$
\left(X_{S_{h} T_{k}} ; 1 \leq h \leq i, 1 \leq k \leq j\right)=_{\mathscr{D}}\left(X_{h k} ; 1 \leq h \leq i, 1 \leq k \leq j\right) .
$$

PROOF. We begin by proving the theorem for a finite array, and assume that both $c, r<\infty$. The extension to the case of an infinite array is discussed at the end of the proof.

(1) This is an immediate consequence of [7, Proposition 5.1] and Lemma 3.1.

(2) (a) Assume that $T_{k}$ is $\mathscr{F}_{0}^{1}$-measurable, for all $k=1, \ldots, j$. Let $f_{h k}: G \rightarrow \mathbb{R}$ be bounded measurable functions, $h=1, \ldots, i ; k=1, \ldots, j$. Then

$$
\begin{aligned}
E\left[\prod_{h=1}^{i} \prod_{k=1}^{j} f_{h k}\left(X_{S_{h} T_{k}}\right)\right] & =E\left[E\left(\prod_{h=1}^{i} \prod_{k=1}^{j} f_{h k}\left(X_{S_{h} T_{k}}\right) \mid \mathscr{F}_{0}^{\prime}\right)\right] \\
& =E\left[E\left(\prod_{h=1}^{i} \prod_{k=1}^{j} f_{h k}\left(X_{h T_{k}}\right) \mid \mathscr{F}_{0}^{\prime}\right)\right] \\
& =E\left[\prod_{h=1}^{i} \prod_{k=1}^{j} f_{h k}\left(X_{h T_{k}}\right)\right] \\
& =E\left[\prod_{h=1}^{i} \prod_{k=1}^{j} f_{h k}\left(X_{h k}\right)\right] .
\end{aligned}
$$

We apply the predictable sampling theorem for spreadable sequences ([7, Proposition 5.1]) and (23) follows from the $\mathscr{F}^{1}$-spreadability of the column vectors and the fact that, given $\mathscr{F}_{0}^{1}$, the rows become fixed. Equality (24) then follows from the $\mathscr{F}^{2}$-spreadability of the row vectors. A monotone class argument completes the proof. 
(2) (b) Let $f_{h k}: G \rightarrow \mathbb{R}$ be bounded and measurable, $1 \leq h \leq i, 1 \leq k \leq j$. By symmetry, we may assume without loss of generality that $i \leq j$. Applying conditional spreadability iteratively,

$$
\begin{aligned}
E\left[\prod_{h=1}^{i} \prod_{k=1}^{j} f_{h k}\left(X_{S_{h} T_{k}}\right)\right] \\
=\sum_{\substack{1 \leq a_{1}<\ldots<a_{i} \leq c \\
1 \leq b_{1}<\ldots<b_{j} \leq r}} E\left[\prod_{h=1}^{i} \prod_{k=1}^{j} f_{h k}\left(X_{S_{h}, T_{k}}\right) ; \begin{array}{l}
S_{1}=a_{1}, \ldots, S_{i}=a_{i}, \\
T_{1}=b_{1}, \ldots, T_{j}=b_{j}
\end{array}\right] \\
=\sum_{\substack{1 \leq a_{1}<\ldots<a_{i} \leq c \\
1 \leq b_{1}<\ldots<b_{j} \leq r}} E\left[E\left(f_{i j}\left(X_{a_{i} b_{j}}\right) \prod_{h=1}^{i-1} f_{h j}\left(X_{a_{h} b_{j}}\right) \prod_{k=1}^{j-1} f_{i k}\left(X_{a_{i} b_{k}}\right) \mid \mathscr{F}_{a_{i}-1, b_{j}-1}\right)\right. \\
\left.\quad \times \prod_{h=1}^{i-1 j-1} \prod_{k=1}^{j-1} f_{h k}\left(X_{a_{h} b_{k}}\right) ; \begin{array}{l}
S_{1}=a_{1}, \ldots, S_{i}=a_{i}, \\
T_{1}=b_{1}, \ldots, T_{j}=b_{j}
\end{array}\right]
\end{aligned}
$$

$$
\begin{aligned}
= & \sum_{\substack{1 \leq a_{1}<\cdots<a_{i} \leq c \\
1 \leq b_{1}<\cdots<b_{j} \leq r}} E\left[E\left(f_{i j}\left(X_{c r}\right) \prod_{h=1}^{i-1} f_{h j}\left(X_{a_{h}}\right) \prod_{k=1}^{j-1} f_{i k}\left(X_{c b_{k}}\right) \mid \mathscr{F}_{a_{i}-1, b_{j}-1}\right)\right. \\
& \left.\times \prod_{h=1}^{i-1} \prod_{k=1}^{j-1} f_{h k}\left(X_{a_{h} b_{k}}\right) ; \begin{array}{l}
S_{1}=a_{1}, \ldots, S_{i}=a_{i}, \\
T_{1}=b_{1}, \ldots, T_{j}=b_{j}
\end{array}\right] \\
= & \sum_{\substack{1 \leq a_{1}<\cdots<a_{i-1} \leq c-1 \\
1 \leq b_{1}<\cdots<b_{j-1} \leq r-1}} E\left[f_{i j}\left(X_{c r}\right) \prod_{h=1}^{i-1} f_{h j}\left(X_{S_{h} r}\right) \prod_{k=1}^{j-1} f_{i k}\left(X_{c T_{k}}\right)\right.
\end{aligned}
$$$$
\left.\times \prod_{h=1}^{i-1} \prod_{k=1}^{j-1} f_{h k}\left(X_{S_{h} T_{k}}\right) ; \begin{array}{l}
S_{1}=a_{1}, \ldots, S_{i-1}=a_{i-1} \\
T_{1}=b_{1}, \ldots, T_{j-1}=b_{j-1}
\end{array}\right]
$$

$$
\begin{aligned}
= & \sum_{\substack{1 \leq a_{1}<\ldots<a_{-2} \leq c-2 \\
1 \leq b_{1}<\cdots<b_{j-2} \leq r-2}} E\left[f_{i j}\left(X_{c r}\right) f_{i-1, j}\left(X_{c-1, r}\right) f_{i, j-1}\left(X_{c, r-1}\right) f_{i-1, j-1}\left(X_{c-1, r-1}\right)\right. \\
& \times \prod_{h=1}^{i-2} f_{h, j-1}\left(X_{S_{h}, r-1}\right) f_{h j}\left(X_{S_{h} r}\right) \prod_{k=1}^{j-2} f_{i-1, k}\left(X_{c-1, T_{k}}\right) f_{i k}\left(X_{c T_{k}}\right) \\
& \left.\times \prod_{h=1}^{i-2} \prod_{k=1}^{j-2} f_{h k}\left(X_{S_{h} T_{k}}\right) ; \begin{array}{l}
S_{1}=a_{1}, \ldots, S_{i-2}=a_{i-2}, \\
T_{1}=b_{1}, \ldots, T_{j-2}=b_{j-2}
\end{array}\right]
\end{aligned}
$$

$$
\begin{aligned}
= & \cdots=\sum_{\substack{1 \leq a_{1} \leq c-i+1 \\
1 \leq b_{1}<\cdots<b_{j-i+1} \leq r-i+1}} E\left[\prod_{k=1}^{j-i+1} f_{1 k}\left(X_{S_{1} T_{k}}\right) \prod_{h=2}^{i} \prod_{k=1}^{j-i+1} f_{h k}\left(X_{c-i+h, T_{k}}\right)\right. \\
& \left.\times \prod_{k=j-i+2}^{j} f_{1 k}\left(X_{S_{1}, r-j+k}\right) \prod_{h=2}^{i} \prod_{k=j-i+2}^{j} f_{h k}\left(X_{c-i+h, r-j+k}\right) ; \begin{array}{l}
S_{1}=a_{1}, T_{1}=b_{1}, \ldots, \\
T_{j-i+1}=b_{j-i+1}
\end{array}\right]
\end{aligned}
$$


(28)

$$
\begin{aligned}
= & \sum_{\substack{1 \leq a_{1} \leq c-i+1 \\
1 \leq b_{1}<\cdots<b_{j-i+1} \leq r-i+1}} E\left[E \left(f_{1, j-i+1}\left(X_{a_{1} b_{j-i+1}}\right) \prod_{k=1}^{j-i} f_{1 k}\left(X_{a_{1} b_{k}}\right)\right.\right. \\
& \times \prod_{h=2}^{i} f_{h, j-i+1}\left(X_{c-i+h, b_{j-i+1}}\right) \prod_{h=2}^{i} \prod_{k=1}^{j-i} f_{h k}\left(X_{c-i+h, b_{k}}\right) \prod_{k=j-i+2}^{j} f_{1 k}\left(X_{a_{1}, r-j+k}\right) \\
& \left.\left.\times \prod_{h=2}^{i} \prod_{k=j-i+2}^{j} f_{h k}\left(X_{c-i+h, r-j+k}\right) \mid \mathscr{F}_{a_{1}-1, b_{j-i+1}-1}\right) ; \begin{array}{l}
S_{1}=a_{1}, T_{1}=b_{1}, \ldots, \\
T_{j-i+1}=b_{j-i+1}
\end{array}\right]
\end{aligned}
$$

$$
\begin{aligned}
= & \sum_{1 \leq b_{1}<\cdots<b_{j-i} \leq r-i} E\left[\prod_{h=1}^{i} \prod_{k=1}^{j-i} f_{h k}\left(X_{c-i+h, T_{k}}\right)\right. \\
& \left.\times \prod_{h=1}^{i} \prod_{k=j-i+1}^{j} f_{h k}\left(X_{c-i+h, r-j+k}\right) ; T_{1}=b_{1}, \ldots, T_{j-i}=b_{j-i}\right] \\
= & \sum_{1 \leq b_{1}<\cdots<b_{j-i} \leq r-i} E\left[E \left(\prod_{h=1}^{i} f_{h, j-i}\left(X_{c-i+h, b_{j-i}}\right) \prod_{h=1}^{i} \prod_{k=1}^{j-i-1} f_{h k}\left(X_{c-i+h, b_{k}}\right)\right.\right. \\
& \left.\left.\times \prod_{h=1}^{i} \prod_{k=j-i+1}^{j} f_{h k}\left(X_{c-i+h, r-j+k}\right) \mid \mathscr{F}_{b_{j-i}-1}^{2}\right) ; T_{1}=b_{1}, \ldots, T_{j-i}=b_{j-i}\right]
\end{aligned}
$$

$$
\begin{aligned}
= & \sum_{1 \leq b_{1}<\ldots<b_{j-i-1} \leq r-i-1} E\left[\prod_{h=1}^{i} \prod_{k=1}^{j-i-1} f_{h k}\left(X_{c-i+h, T_{k}}\right)\right. \\
& \left.\times \prod_{h=1}^{i} \prod_{k=j-i}^{j} f_{h k}\left(X_{c-i+h, r-j+k}\right) ; T_{1}=b_{1}, \ldots, T_{j-i-1}=b_{j-i-1}\right] \\
= & \ldots=\sum_{1 \leq b_{1} \leq r-j+1} E\left[\prod_{h=1}^{i} f_{h 1}\left(X_{c-i+h, T_{1}}\right) \prod_{h=1}^{i} \prod_{k=2}^{j} f_{h k}\left(X_{c-i+h, r-j+k}\right) ; T_{1}=b_{1}\right] \\
= & \sum_{1 \leq b_{1} \leq r-j+1} E\left[E \left(\prod_{h=1}^{i} f_{h 1}\left(X_{c-i+h, b_{1}}\right)\right.\right. \\
& \left.\left.\times \prod_{h=1}^{i} \prod_{k=2}^{j} f_{h k}\left(X_{c-i+h, r-j+k}\right) \mid \mathscr{F}_{b_{1}-1}^{2}\right) ; T_{1}=b_{1}\right] \\
= & \sum_{1 \leq b_{1} \leq r-j+1} E\left[\prod_{h=1}^{i} \prod_{k=1}^{j} f_{h k}\left(X_{c-i+h, r-j+k}\right) ; T_{1}=b_{1}\right] \\
= & E\left[\prod_{h=1}^{i} \prod_{k=1}^{j} f_{h k}\left(X_{c-i+h, r-j+k}\right)\right]=E\left[\prod_{h=1}^{i} \prod_{k=1}^{j} f_{h k}\left(X_{h k}\right)\right] .
\end{aligned}
$$

Conditional separate spreadability given $\mathscr{F}_{a_{i}-1, b_{j}-1}$ is used to obtain (25); (26) is 
obtained by a similar argument conditioning on $\mathscr{F}_{a_{i-1}-1, b_{j-1}-1}$ and then adding over $a_{i-1}$ and $b_{j-1}$. We continue to apply separate spreadability and condition successively on $\mathscr{F}_{a_{i-2}-1, b_{j-2}-1}, \ldots, \mathscr{F}_{a_{2}-1, b_{j-i+2}-1}$ to obtain (27) and next on $\mathscr{F}_{a_{1}-1, b_{j-i+1}-1}$ to obtain (28) and (29). We now have to deal only with spreadability of the row vectors to obtain (30) and (31). One last application of separate spreadability yields (32). The proof is completed with a monotone class argument.

All the above cases can be extended to infinite arrays and bounded sequences of predictable times by the simple observation that any finite subarray of an infinite $\mathscr{F}$-SS array is $\mathscr{F}$-SS. Finally, to extend the preceding results to general sequences of predictable times, as in the proof of [8, Theorem 11.13], introduce the bounded $\mathscr{F}^{1}$ and $\mathscr{F}^{2}$-predictable times

$$
\begin{array}{ll}
S_{h}^{m}=S_{h} I\left\{S_{h} \leq m\right\}+(m+h) I\left\{S_{h}>m\right\}, & h=1, \ldots, i \quad \text { and } \\
T_{k}^{m}=T_{k} I\left\{T_{k} \leq m\right\}+(m+k) I\left\{T_{k}>m\right\}, & k=1, \ldots, j .
\end{array}
$$

Clearly, $\left(S_{h}^{m}, T_{k}^{m}\right)$ is $\mathscr{F}$-predictable if $\left(S_{h}, T_{k}\right)$ is, and so in all cases we may let $m \rightarrow \infty$ and (20), (21) and (22) follow.

COMMENT 3.5. In the proof of (2) (b) above, $\mathscr{F}$-predictability was required only for the pairs $\left(S_{1}, T_{j-i+1}\right),\left(S_{2}, T_{j-i+2}\right), \ldots,\left(S_{i}, T_{j}\right)$. However, the more general statement in the theorem allows an immediate extension to infinite arrays and infinite increasing sequences $\left(S_{1}, S_{2}, \ldots\right)$ and $\left(T_{1}, T_{2}, \ldots\right)$ of finite $\mathscr{F}^{1}$ - and $\mathscr{F}^{2}$-predictable stopping times. In this case, the statement of the theorem holds for every pair of finite subsequences $\left(S_{1}, \ldots, S_{i}\right)$ and $\left(T_{1}, \ldots, T_{j}\right)$.

3.2. Weak $\mathscr{F}$-separately spreadable arrays We now turn our attention to weak $\mathscr{F}$-SS arrays. From (11), $X$ is weak $\mathscr{F}$-SS if conditioned on $\mathscr{F}_{j k}, \theta_{j k} \circ X$ is SS for all $j<c$, and all $k<r$. As pointed out in Section 2, weak $\mathscr{F}$-SS does not imply $\mathscr{F}$-SS.

In this context, we say that the array $X$ is $\mathscr{F}$-stationary if $\theta_{S T} \circ X=_{\mathscr{D}} X$ for every bounded adapted random time $(S, T)$. For $c, r<\infty$, this is interpreted as

$$
\left(X_{h k} ; S+1 \leq h \leq S+l, T+1 \leq k \leq T+m\right)=_{\mathscr{D}}\left(X_{h k} ; 1 \leq h \leq l, 1 \leq k \leq m\right)
$$

whenever $S+l \leq c$ and $T+m \leq r$ a.s.

Next, we consider the two-parameter prediction sequence

$$
\mu_{i j}=P\left(\theta_{i j} \circ X \in \cdot \mid \mathscr{F}_{i j}\right) .
$$

We say that $\mu_{i j}$ forms an $\mathscr{F}$-martingale if for all $h \geq i, k \geq j, \theta_{h k} \circ X=\theta_{i j} \circ X$ over $\mathscr{F}_{i j}$ for all $i, j \geq 0$, that is, for $A \in \mathscr{F}_{i j}, i, j \geq 0$,

$$
P\left[\theta_{h k} \circ X \in ; A\right]=P\left[\theta_{i j} \circ X \in ; A\right] \text {. }
$$


For finite arrays, this condition is interpreted as: for $1 \leq i \leq h \leq c-1,1 \leq j \leq k \leq$ $r-1$

$$
\begin{aligned}
& \left(X_{l m} ; h+1 \leq l \leq c, k+1 \leq m \leq r\right) \\
& \quad=\mathscr{g}\left(X_{l m} ; i+1 \leq l \leq c-h+i, j+1 \leq m \leq r-k+j\right) \quad \text { over } \mathscr{F}_{i j} .
\end{aligned}
$$

LEMMA 3.6. Let $X$ be a finite or infinite array. The following are equivalent:

(1) $X$ is a weak $\mathscr{F}$-SS array.

(2) $X$ is $\mathscr{F}$-stationary.

(3) $\mu_{i j}$ forms an $\mathscr{F}$-martingale.

PROOF. $1 \Rightarrow 2$ : If $X$ is an infinite weak $\mathscr{F}$-SS array, the proof that $X$ is $\mathscr{F}$. stationary is identical to that of $[4$, Theorem 1$]$ noting that only spreadability is required.

$2 \Rightarrow 3$ : By $[4$, Lemma 1], it is enough to show for any bounded $\mathscr{F}$-adapted time $(S, T)$ that $E\left[\mu_{S T}\right]=E\left[\mu_{00}\right]$.

$$
\begin{aligned}
E\left[\mu_{S T}\right] & =\sum_{i} \sum_{j} E\left[\mu_{i j} ; S=i, T=j\right] \\
& =\sum_{i} \sum_{j} E\left[P\left(\theta_{i j} \circ X \in \cdot \mid \mathscr{F}_{i j}\right) ; S=i, T=j\right] \\
& =\sum_{i} \sum_{j} E\left[E\left(I\left(\theta_{S T} \circ X \in \cdot ; S=i, T=j\right) \mid \mathscr{F}_{i j}\right)\right] \\
& =P\left[\theta_{S, T} \circ X \in \cdot\right] \\
& =P[X \in \cdot] \\
& =E\left[\mu_{00}\right] .
\end{aligned}
$$

(33) is a result of $\mathscr{F}$-stationarity, and so the martingale property follows.

$3 \Rightarrow 1$ : A simple argument by induction shows that $X$ is weak $\mathscr{F}$-SS if for $0 \leq i \leq c$, $0 \leq j \leq r$,

$$
\begin{aligned}
& P\left[\theta_{i+1, j} \circ X \in \cdot \mid \mathscr{F}_{i j}\right]=P\left[\theta_{i j} \circ X \in \cdot \mid \mathscr{F}_{i j}\right] \text { and } \\
& P\left[\theta_{i, j+1} \circ X \in \cdot \mid \mathscr{F}_{i j}\right]=P\left[\theta_{i j} \circ X \in \cdot \mid \mathscr{F}_{i j}\right]
\end{aligned}
$$

It is easily seen that (34) and (35) are immediate from the martingale property.

COMMENT 3.7. Here, we cannot readily express the martingale condition in the preceding lemma in terms of the deletion operator. As pointed out in Section 3.1, for a sequence, from a distributional point of view the shift operator $\theta_{i}$ and deletion operator $\phi_{i}$ are essentially equivalent, given $\mathscr{F}_{i-1}$. This is not true for an array $X$. Given $\mathscr{F}_{i j}$, the distribution of $\theta_{i j} \circ X$ involves only the shifted array, whereas the distribution of $\phi_{i+1, j+1} \circ X$ involves the entire shell region. 
We come to a predictable sampling theorem for weak $\mathscr{F}$-SS arrays.

THEOREM 3.8. Suppose that $X$ is a finite or infinite weak $\mathscr{F}$-SS array and that $(1,1) \leq\left(S_{1}, T_{1}\right)<\cdots<\left(S_{n}, T_{n}\right) \leq(c, r)$ are finite $\mathscr{F}$-predictable random times. Then $\left(X_{S_{1} T_{1}}, \ldots, X_{S_{n} T_{n}}\right)=_{\mathscr{D}}\left(X_{11}, \ldots, X_{n n}\right)$.

ProOf. In what follows, we assume that $X$ is a finite $c \times r$ array. Then we may use the same argument as in the proof of Theorem 3.4 to extend the result to an infinite array.

Let $(S, T)$ be a predictable random time. For a bounded measurable function $f: G \rightarrow \mathbb{R}$,

$$
\begin{aligned}
E\left[f\left(X_{S T}\right)\right] & =\sum_{i=1}^{c} \sum_{j=1}^{r} E\left[f\left(X_{i j}\right) ;(S, T)=(i, j)\right] \\
& =\sum_{i=1}^{c} \sum_{j=1}^{r} E\left[f\left(X_{c r}\right) ;(S, T)=(i, j)\right] \\
& =E\left[f\left(X_{c r}\right)\right]=E\left[f\left(X_{11}\right)\right] .
\end{aligned}
$$

(36) follows by weak $\mathscr{F}$-spreadability and the fact that $\{(S, T)=(i, j)\} \in \mathscr{F}_{i-1, j-1}$.

As in the proof of [7, Theorem 5.1], we proceed by induction on $n$. Assuming that the statement of the theorem is true for increasing sequences of $n-1$ predictable times, for $f_{h}: G \rightarrow \mathbb{B}$ bounded and measurable, $h=1, \ldots, n$,

$$
\begin{aligned}
E\left[\prod_{h=1}^{n} f_{h}\left(X_{S_{h} T_{h}}\right)\right] & =\sum_{i=1}^{c-n+1} \sum_{j=1}^{r-n+1} E\left[f_{1}\left(X_{i j}\right) \prod_{h=2}^{n} f_{h}\left(X_{S_{h} T_{h}}\right) ;\left(S_{1}, T_{1}\right)=(i, j)\right] \\
& =\sum_{i=1}^{c-n+1} \sum_{j=1}^{r-n+1} E\left[f_{1}\left(X_{i j}\right) \prod_{h=2}^{n} f_{h}\left(X_{i+h-1, j+h-1}\right) ;\left(S_{1}, T_{1}\right)=(i, j)\right] \\
& =\sum_{i=1}^{c-n+1} \sum_{j=1}^{r-n+1} E\left[\prod_{h=1}^{n} f_{h}\left(X_{c-n+h, r-n+h}\right) ;\left(S_{1}, T_{1}\right)=(i, j)\right] \\
& =E\left[\prod_{h=1}^{n} f_{h}\left(X_{c-n+h, r-n+h}\right)\right] \\
& =E\left[\prod_{h=1}^{n} f_{h}\left(X_{h, h}\right)\right] .
\end{aligned}
$$

To prove (37), the induction hypothesis is applied to the array $\left(f_{1}\left(X_{i j}\right), \theta_{i j} \circ X\right)$, which is conditionally spreadable, given $\mathscr{F}_{i j}$. Next, (38) follows from the fact that given $\mathscr{F}_{i-1, j-1}, \theta_{i-1, j-1} \circ X$ is spreadable. Finally, (39) follows from the spreadability of $X$. 
COMMENT 3.9. We note that the statement of Theorem 3.8 is much weaker than that of Theorem 3.4, in that the predictable times must be strictly increasing in the partial order on $\mathbb{Z}_{+}^{2}$. The reason for this is that the weak $\mathscr{F}$-SS property is based on successive shifts, and so it is not possible to deal with random times that are incomparable with respect to the partial order. As discussed in Section 2, such incomparable points would lie in shoulder regions with respect to some $\sigma$-fields, and the behaviour is not determined by the weak $\mathscr{F}$-SS property.

\section{Joint spreadability}

4.1. Strong $\mathscr{F}$-jointly spreadable arrays We now focus on square $n \times n$ arrays ( $n$ may be $\infty$ ) and perform the same operations on the rows and columns. $X$ is strong $\mathscr{F}$-JS, or just $\mathscr{F}$-JS, if for every $i<j, k \leq n ; j, k<\infty, \phi_{j j} \circ X=\mathscr{D P}_{\mathscr{F}_{i i}} \phi_{k k} \circ X$. We observe that if $X$ is JS, then all of the diagonal elements must have the same distribution. Likewise, the random variables $\left(X_{i j} ; i<j\right)$ must be identically distributed, and the random variables $\left(X_{i j} ; j<i\right)$ must also be identically distributed. However, the three distributions can be different.

For the martingale characterization of $\mathscr{F}$-JS arrays, we define only one (1-dimensional) prediction sequence $\mu_{k}=P\left[\phi_{k+1, k+1} \circ X \in \cdot \mid \mathscr{F}_{k k}\right]$.

Here we note that we must use the deletion operator rather than the shift operator in order to control the behaviour of the entire shell, rather than simply the shifted array (see Comment 3.7).

We say that $\mu_{i}$ forms an $\mathscr{F}_{i i}$-martingale if for all $n \geq h \geq i \geq 1, \phi_{h h} \circ X={ }_{\mathscr{D}} \phi_{i i} \circ X$ over $\mathscr{F}_{i-1, i-1}$.

LEMMA 4.1. Let $X$ be an $n \times n$ array $(n$ may be $\infty)$. The following are equivalent:

(1) $X$ is $\mathscr{F}-J S$.

(2) For any finite $m \leq n$ and any $\mathscr{F}_{i i}$-predictable random time $\tau$ such that $\tau \leq m<$ $\infty$ a.s., then $\phi_{\tau \tau} \circ X=\mathscr{D} \phi_{m m} \circ X$.

(3) $\mu_{i}$ is an $\mathscr{F}_{i i}$-martingale.

PROOF. $1 \Rightarrow 2$ : Let $X$ be $\mathscr{F}$-JS and assume $\tau$ is $\mathscr{F}_{i i}$-predictable and bounded a.s. by $m$. Then for $f: G^{2(n-1)} \rightarrow \mathbb{R}$ a measurable and bounded function, by conditional spreadability given $\mathscr{F}_{i-1, i-1}$,

$$
\begin{aligned}
E\left[f\left(\phi_{\tau \tau} \circ X\right)\right] & =\sum_{i=1}^{m} E\left[f\left(\phi_{\tau \tau} \circ X\right) ; \tau=i\right] \\
& =\sum_{i=1}^{m} E\left[E\left(f\left(\phi_{i i} \circ X\right) \mid \mathscr{F}_{i-1, i-1}\right) ; \tau=i\right]
\end{aligned}
$$




$$
\begin{aligned}
& =\sum_{i=1}^{m} E\left[E\left(f\left(\phi_{m m} \circ X\right) \mid \mathscr{F}_{i-1, i-1}\right) ; \tau=i\right] \\
& =E\left[f\left(\phi_{m m} \circ X\right)\right] .
\end{aligned}
$$

The result follows by a monotone class argument.

$2 \Rightarrow 3:$ Let $A \in \mathscr{F}_{i-1, i-1}$ for some $1 \leq i \leq n$ and let $h \geq i$. Define

$$
\tau= \begin{cases}i & \text { on } A \\ h & \text { on } A^{c}\end{cases}
$$

It follows that $\tau$ is $\mathscr{F}_{i i}$-predictable, and so for $f: G^{2(n-1)} \rightarrow \mathbb{B}$ bounded and measurable,

$$
\begin{aligned}
& E\left[f\left(\phi_{\tau \tau} \circ X\right)\right]=E\left[f\left(\phi_{h h} \circ X\right)\right] \\
& \quad \Rightarrow \int_{A} f\left(\phi_{i i} \circ X\right) d P+\int_{A^{c}} f\left(\phi_{h h} \circ X\right) d P=\int_{\Omega} f\left(\phi_{h h} \circ X\right) d P \\
& \quad \Rightarrow \int_{A} f\left(\phi_{i i} \circ X\right) d P=\int_{A} f\left(\phi_{h h} \circ X\right) d P .
\end{aligned}
$$

Therefore, by a monotone class argument, on $\mathscr{F}_{i-1, i-1}, \phi_{h h} \circ X=\mathscr{D} \phi_{i i} \circ X$.

$3 \Rightarrow 1$ : Since $\mu_{i}$ is an $\mathscr{F}_{i i}$-martingale, for $i<j<k \leq n ; j, k<\infty$,

$$
\begin{aligned}
P\left[\phi_{k k} \circ X \in \cdot \mid \mathscr{F}_{i i}\right] & =E\left(P\left[\phi_{k k} \circ X \in \cdot \mid \mathscr{F}_{k-1, k-1}\right] \mid \mathscr{F}_{i i}\right] \\
& =E\left[\mu_{k-1} \mid \mathscr{F}_{i i}\right]=E\left[\mu_{j-1} \mid \mathscr{F}_{i i}\right]=P\left[\phi_{j j} \circ X \in \cdot \mid \mathscr{F}_{i i}\right]
\end{aligned}
$$

This is the $\mathscr{F}$-JS property.

We have the following predictable sampling theorem. The proof is omitted, as the method is similar to that used to prove Theorem 3.4. Note that in the case of joint spreadability, both components of the random times must come from a single sequence of (one dimensional) predictable times.

THEOREM 4.2. Let $X$ be $a \times n$ ( $n$ may be $\infty$ ) $\mathscr{F}-J S$ array and suppose that $1 \leq \tau_{1}<\cdots<\tau_{k} \leq n$ is a sequence of finite $\mathscr{F}_{i i}$-predictable times. Then

$$
\left(X_{\tau_{i} \tau_{j}} ; 1 \leq i, j \leq k\right)=_{\mathscr{D}}\left(X_{i j} ; 1 \leq i, j \leq k\right)
$$

4.2. Weak $\mathscr{F}$-jointly spreadable arrays Turning to the weak $\mathscr{F}$ property, the $n \times n$ array $X$ is weak $\mathscr{F}$-JS if conditioned on $\mathscr{F}_{k k}, \theta_{k k} \circ X$ is JS, for all $k<n$. Here we have a characterization of the weak $\mathscr{F}$-JS property in terms of a (two parameter) martingale, but we shall see that the martingale must be defined by conditioning on a different filtration $\mathscr{H}$ that is essentially one-dimensional. 
First, we begin with a weaker but simpler result: we say that $X$ is $\mathscr{F}$-jointly stationary if $\theta_{\tau \tau} \circ X=\mathscr{D} X$ for every bounded $\mathscr{F}_{i i}$-adapted random time $\tau$. For $n<\infty$, this is interpreted as $\left\{X_{h k} ; \tau+1 \leq h, k \leq \tau+l\right\}=_{\mathscr{D}}\left\{X_{h k} ; 1 \leq h, k \leq l\right\}$ whenever $\tau+l \leq n$ a.s.

Next, define $\mu_{i}=P\left[\theta_{i i} \circ X \in \cdot \mid \mathscr{F}_{i i}\right]$. As before, $\mu_{i}$ forms an $\mathscr{F}_{i i}$-martingale if for all $l \geq i, \theta_{l l} \circ X=_{\mathscr{D}} \theta_{i i} \circ X$ over $\mathscr{F}_{i i}$. For finite arrays, we interpret this as: for $1 \leq i \leq l \leq n-1,\left\{X_{h k} ; l+1 \leq h, k \leq n\right\}=\mathscr{D}\left\{X_{h k} ; i+1 \leq h, k \leq n-l+i\right\}$ over $\mathscr{F}_{i i}$.

LEMMA 4.3. Let $X$ be a finite or infinite $n \times n$ weak $\mathscr{F}-J S$ array. Then

(1) $X$ is $\mathscr{F}$-jointly stationary;

(2) $\mu_{i}$ forms an $\mathscr{F}_{i i}$-martingale.

PROOF. It is easily seen that (1) and (2) are equivalent, so we will show that $\mathscr{F}$ joint stationarity is implied by weak $\mathscr{F}$-joint spreadability. If $X$ is weak $\mathscr{F}$-JS and $\tau \leq m-k$ a.s., $(m \leq n, m<\infty)$ and if $f_{i j}: G \rightarrow \mathbb{R}$ is bounded and measurable for all $1 \leq i, j \leq k$, then

$$
\begin{aligned}
& E\left[\prod_{i=1}^{k} \prod_{j=1}^{k} f_{i j}\left(X_{\tau+i, \tau+j}\right)\right] \\
& \quad=\sum_{h=0}^{m-k} E\left[\prod_{i=1}^{k} \prod_{j=1}^{k} f_{i j}\left(X_{\tau+i, \tau+j}\right) ; \tau=h\right] \\
& \quad=\sum_{h=0}^{m-k} E\left[E\left(\prod_{i=1}^{k} \prod_{j=1}^{k} f_{i j}\left(X_{h+i, h+j}\right) \mid \mathscr{F}_{h h}\right) ; \tau=h\right] \\
& \quad=\sum_{h=0}^{m-k} E\left[E\left(\prod_{i=1}^{k} \prod_{j=1}^{k} f_{i j}\left(X_{m-k+i, m-k+j}\right) \mid \mathscr{F}_{h h}\right) ; \tau=h\right] \\
& \quad=E\left[\prod_{i=1}^{k} \prod_{j=1}^{k} f_{i j}\left(X_{m-k+i, m-k+j}\right)\right]=E\left[\prod_{i=1}^{k} \prod_{j=1}^{k} f_{i j}\left(X_{i j}\right)\right] .
\end{aligned}
$$

Once again, a monotone class argument proves $\mathscr{F}$-joint stationarity.

COMMENT 4.4. It is not true that (1) and (2) of Lemma 4.3 imply that $X$ is weak $\mathscr{F}$-JS, as the following deterministic counterexample shows: for $a$ and $b$ distinct real numbers, let

$$
X=\left[\begin{array}{lll}
b & a & b \\
a & b & a \\
b & a & b
\end{array}\right]
$$


Here $X$ is jointly stationary but not jointly spreadable (consider the array after deletion of the second row and column).

In order to understand the more complicated martingale structure that is required for weak $\mathscr{F}$-joint spreadability, we observe that in Lemma 4.1, the (one-dimensional) martingale was defined via symmetric deletions, while in Lemma 3.6, the (twodimensional) martingale was defined in terms of shifts in the rows and columns separately. Here, a combination of a shift followed by a deletion (both one-dimensional) is needed to determine the weak $\mathscr{F}$-JS property. If only deletions are used, the entire shell is affected. If a (symmetric) shift $\theta_{i i}$ is used, the effect will be to delete all the first $i$ rows and columns. As seen in the counterexample above, if $i>1$, it is not possible to delete, say, only the $i^{\text {th }}$ row and column using symmetric shifts. In fact, the $n \times n$ ( $n$ may be $\infty$ ) array $X$ is weak $\mathscr{F}$-JS if and only if for every $i \leq n$ and $j, k \leq n-i$, where $i, j, k<\infty$,

$$
\phi_{j j} \circ \theta_{i i} \circ X=\phi_{j j} \circ\left(\theta_{i i} \circ X\right)=\mathscr{D P F}_{i i} \phi_{k k} \circ\left(\theta_{i i} \circ X\right)=\phi_{k k} \circ \theta_{i i} \circ X .
$$

It is the combination of the shift and the deletion that will define the indices of the martingale, but only the index associated with the shift is used to determine the conditioning in the definition of the martingale. With this in mind, we observe that the weak $\mathscr{F}$-JS property also implies, for every $i \leq l \leq n$ and $j, k \leq n-l$, where $i, j, k, l<\infty$,

$$
\phi_{j j} \circ \theta_{i i} \circ X={\mathscr{D} \mid \mathscr{F}_{i i}}_{k k} \circ \theta_{l l} \circ X .
$$

(This is interpreted in the obvious way for finite arrays:

$$
\begin{aligned}
\left(X_{a b}\right. & : a, b \in\{i+1, \ldots, i+j-1, i+j+1, \ldots, n-(l-i)\}) \\
& ={ }_{\mathscr{D} \mid \mathscr{F}_{i i}}\left(X_{a b}: a, b \in\{l+1, \ldots, l+k-1, l+k+1, \ldots, n\}\right) .
\end{aligned}
$$

This follows as the right-hand side of (40) is $\theta_{i i} \circ X$ with the first $l-i$ rows and columns deleted, as well as the $(l-i+k)^{\text {th }}$ row and column deleted.

Let $\mathscr{H}_{i j}=\mathscr{F}_{i i}$, for all $i, j$. This is a 2-dimensional filtration that is really just 1-dimensional. We note that $X$ is not $\mathscr{H}$-adapted, and so in what follows, although we will be making use of $\mathscr{H}$-adapted random times $(S, T)$ (that is, $\{S=i$, $T=j\} \in \mathscr{H}_{i j}=\mathscr{F}_{i i}$, for all $\left.i, j\right)$, we do not use the term $\mathscr{H}$-stationary in describing the behaviour of $X$.

Now, let $\mu_{l k}=P\left[\phi_{k k} \circ \theta_{l l} \circ X \in \cdot \mid \mathscr{H}_{l k}\right], 0 \leq l \leq n-1,0 \leq k \leq n$, and $k, l<\infty$. Here $\mu_{l k}$ is an $\mathscr{H}$-martingale if for all $(l, k) \geq(i, j)$ and for all $A \in \mathscr{H}_{i j}\left(=\mathscr{F}_{i i}\right)$,

$$
P\left[\phi_{k k} \circ \theta_{l l} \circ X \in ; A\right]=P\left[\phi_{j j} \circ \theta_{i i} \circ X \in ; A\right] .
$$

The martingale condition is interpreted as in (41) for finite arrays.

We now have a martingale characterization of the weak $\mathscr{F}$-JS property: 
LEMMA 4.5. Let $X$ be a finite or infinite $n \times n$ array. The following are equivalent:

(1) $X$ is weak $\mathscr{F}$-JS;

(2) $\phi_{T T} \circ \theta_{S S} \circ X=\mathscr{D} \phi_{k k} \circ \theta_{l l} \circ X$ for all $\mathscr{H}$-adapted bounded random times $(S, T)$ with $S \leq l<\infty, T \leq k<\infty, l+k \leq n$ a.s.;

(3) $\mu_{l k}$ is an $\mathscr{H}$-martingale.

Proof. $1 \Rightarrow 2$ : Assume that $X$ is weak $\mathscr{F}$-JS and let $(S, T)$ be $\mathscr{H}$-adapted with $S \leq l$ and $T \leq k$ a.s. Let $f: G^{2(n-l-1)} \rightarrow \mathbb{R}$ be a measurable and bounded function. Using (40), we obtain

$$
\begin{aligned}
E\left[f\left(\phi_{T T} \circ \theta_{S S} \circ X\right)\right] & =\sum_{i=0}^{l} \sum_{j=1}^{k} E\left[E\left(f\left(\phi_{j j} \circ \theta_{i i} \circ X\right) \mid \mathscr{F}_{i i}\right) ; S=i, T=j\right] \\
& =\sum_{i=0}^{l} \sum_{j=1}^{k} E\left[E\left(f\left(\phi_{k k} \circ \theta_{l l} \circ X\right) \mid \mathscr{F}_{i i}\right) ; S=i, T=j\right] \\
& =E\left[f\left(\phi_{k k} \circ \theta_{l l} \circ X\right)\right] .
\end{aligned}
$$

Now apply a monotone class argument.

$2 \Rightarrow 3$ : Let $i \leq l \leq n$ and $j, k \leq n-l$, where $i, j, k, l<\infty$. Let $A \in \mathscr{H}_{i j}=\mathscr{F}_{i i}$ and define

$$
(S, T)= \begin{cases}(i, j) & \text { on } A ; \\ (l, k) & \text { on } A^{c} .\end{cases}
$$

Let $f: G^{2(n-l-1)} \rightarrow \mathbb{B}$ be measurable and bounded. From (2),

$$
\begin{aligned}
& E\left[f\left(\phi_{T T} \circ \theta_{S S} \circ X\right)\right]=E\left[f\left(\phi_{k k} \circ \theta_{l l} \circ X\right)\right] \\
& \quad \Rightarrow \int_{A} f\left(\phi_{j j} \circ \theta_{i i} \circ X\right) d P+\int_{A^{c}} f\left(\phi_{k k} \circ \theta_{l l} \circ X\right) d P=\int_{\Omega} f\left(\phi_{k k} \circ \theta_{l l} \circ X\right) d P \\
& \quad \Rightarrow \int_{A} f\left(\phi_{j j} \circ \theta_{i i} \circ X\right) d P=\int_{A} f\left(\phi_{k k} \circ \theta_{l l} \circ X\right) d P .
\end{aligned}
$$

Therefore, on $\mathscr{H}_{i j}, \phi_{j j} \circ \theta_{i i} \circ X=\mathscr{D} \phi_{k k} \circ \theta_{l l} \circ X$.

$3 \Rightarrow 1$ : If $\mu$ is an $\mathscr{H}$-martingale, then by definition, $\phi_{j j} \circ \theta_{i i} \circ X=\mathscr{D}_{\mid \mathscr{F}_{i i}} \phi_{k k} \circ \theta_{i i} \circ X$, and so $X$ is weak $\mathscr{F}$-JS.

Our final predictable sampling theorem is a straightforward application of the predictable sampling theorem for a spreadable sequence.

LEMMA 4.6. Let $X$ be a $n \times n(n$ may be $\infty)$ weak $\mathscr{F}$-JS array. If $1 \leq \tau_{1}<\cdots<$ $\tau_{k} \leq m$ is a sequence of finite $\mathscr{F}_{i i}$-predictable times, then

$$
\left(X_{\tau_{1} \tau_{1}}, \ldots, X_{\tau_{k} \tau_{k}}\right)=_{\mathscr{D}}\left(X_{11}, \ldots, X_{k k}\right) .
$$




\section{Acknowledgement}

The authors wish to acknowledge very constructive comments from Olav Kallenberg and an anonymous referee that have improved the presentation of the results in this paper.

\section{References}

[1] D. J. Aldous, 'Representations for partially exchangeable arrays of random variables', J. Multivariate Anal. 11 (1981), 581-598.

[2] —_, 'Exchangeability and related topics', in: École d'été de probabilitiés de Saint-Flour XIII, Lecture Notes in Math. 1117 (Springer, Berlin, 1985).

[3] D. N. Hoover, 'Relations on probability spaces and arrays of random variables', Preprint, (Institute of Advanced Study, Princeton, 1979).

[4] B. G. Ivanoff and N. C. Weber, 'Some characterizations of partial exchangeability', J. Austral. Math. Soc. (A) 61 (1996), 345-359.

[5] O. Kallenberg, 'Spreadable and predictable sampling in exchangeable sequences and processes', Ann. Probab. 16 (1988), 508-534.

[6] - 'Symmetries on random arrays and set-indexed processes', J. Theoret. Probab. 5 (1992), 727-765.

[7] ——. 'Spreading-invariant sequences and processes on bounded index sets', Probab. Theory Relat. Fields 118 (2000), 211-250.

[8] _. Foundations of modern probability, 2nd edition (Springer, New York, 2002).

[9] J. F. C. Kingman, 'Uses of exchangeability', Ann. Probab. 6 (1978), 183-197.

[10] E. Merzbach, 'Different kinds of two parameter martingales', Israel J. Math. 52 (1985), 193-208.

[11] C. Ryll-Nardzewski, 'On stationary sequences of random variables and the de Finetti's equivalence', Colloq. Math. 4 (1957), 149-156.

Department of Mathematics and Statistics

University of Ottawa

PO Box 450 Station A

Ottawa, Ontario

Canada K1N 6N5

e-mail: givanoff@uottawa.ca
School of Mathematics and Statistics, F07 University of Sydney NSW 2006

Australia e-mail: neville@maths.usyd.edu.au 